\title{
BMJ Open Amyotrophic lateral sclerosis and the innate immune system: protocol for establishing a biobank and statistical analysis plan
}

\author{
Anne-Lene Kjældgaard (D) , ${ }^{1,2}$ Katrine Pilely, ${ }^{2}$ Karsten Skovgaard Olsen, ${ }^{1}$ \\ Anne Øberg Lauritsen, ${ }^{1}$ Stephen Wørlich Pedersen, ${ }^{3}$ Kirsten Møller, ${ }^{1,4}$ \\ Peter Garred ${ }^{2,4}$
}

To cite: Kjældgaard A-L, Pilely K, Olsen KS, et al. Amyotrophic lateral sclerosis and the innate immune system: protocol for establishing a biobank and statistical analysis plan. BMJ Open 2020;10:e037753. doi:10.1136/ bmjopen-2020-037753

- Prepublication history for this paper is available online. To view these files, please visit the journal online (http://dx.doi. org/10.1136/bmjopen-2020037753).

Received 18 February 2020 Revised 04 May 2020 Accepted 29 June 2020
Check for updates

(C) Author(s) (or their employer(s)) 2020. Re-use permitted under CC BY-NC. No commercial re-use. See rights and permissions. Published by BMJ.

For numbered affiliations see end of article.

Correspondence to Dr Anne-Lene Kjældgaard; akja0044@regionh.dk

\section{ABSTRACT}

Introduction Amyotrophic lateral sclerosis (ALS) is a devastating, progressive disease that causes degeneration of the motor neurons leading to paresis of the bulbar and the skeletal musculature. The pathogenesis of ALS remains unknown. We will test the hypothesis that the complement system is involved in the pathophysiology of ALS. This protocol article describes our efforts to establish a national Danish ALS biobank. The primary aim is to obtain biological material from patients with ALS for the current study as well as for future studies.

Methods and analysis We intend to establish an observational ALS biobank; some of the material from this biobank will be used for a prospective, observational case-control study. The participants are patients with ALS, neurologically healthy controls and non-ALS neurological controls. Each participant consents to be interviewed and to donate blood and cerebrospinal fluid to the biobank. Analysis of the complement system will be carried out on the three groups of patients and compared.

Ethics and dissemination The project has been approved by the Committees on Health Research Ethics in the Capital Region of Denmark (Approval number H-16017145) and the Danish Data Protection Agency (file number 2012-58-0004). All results will be published in peer-reviewed, medical journals and presented at scientific conferences.

Trial registration number NCT02869048

\section{INTRODUCTION}

Amyotrophic lateral sclerosis (ALS) is an aggressive disease that causes progressive degeneration of the upper and lower motor neurons leading to severe muscular dystrophy, fasciculations, hyper-reflexia and paresis of the bulbar and skeletal musculature. As the disease inevitably progresses, the patient becomes unable to move, speak, swallow and breathe. In addition to the neuromuscular symptoms, frontotemporal dementia appears in up to $15 \%$ of all patients with ALS. ${ }^{1}$

ALS is a rare disease with an incidence of $1-2 / 100000$ and a prevalence of $4-6 / 100$
Strengths and limitations of this study

- This prospective, observational study ensures a national amyotrophic lateral sclerosis (ALS) biobank which will facilitate many future clinical studies of a rare disease such as ALS.

- Even though ALS is a heterogeneous disease, all patients face a rapid deterioration and severe distress and the design of this study provides recruitment of patients with ALS at an early clinical stage hence facilitating trial participation.

- The diagnosis of ALS is difficult, and patients follow highly variable courses of disease; thus, some patients who have been recruited for the study will eventually be diagnosed with other diseases and need to be excluded from the present study; conversely, some patients who are in the early phase of ALS may be missed because of atypical symptoms and will therefore be recruited in a later phase.

000. In $70 \%$ of all ALS cases, initial symptoms appear in the upper or lower extremities (spinal ALS), in 5\% symptoms initially appears in the truncus (truncal ALS), and in $25 \%$ a bulbar onset (bulbar ALS) is seen. In a systematic review of the global epidemiology of ALS, the median survival time was reported as 24-48 months from symptom onset. ${ }^{2}$

Five per cent to ten per cent of all ALS cases are of the familial type (fALS) which is genetically heterogeneous mainly with a Mendelian inheritance pattern. ${ }^{3}$ In the remaining patients, there is no family history (sporadic ALS (sALS)). ${ }^{3}$

The pathogenesis of ALS remains unknown and presently, there is no effective treatment to stop the progression. The immune system has been hypothesised to be an important, pathophysiological factor. This is based on the fact that immunological reactions appear both in the proximity of the degenerating 
motor neurons in the central nervous system (CNS) and systemically. ${ }^{4}$

The complement system plays a central role in the innate immune system. The complement system initiates inflammation and activates innate as well as adaptive immune cells by detecting invading pathogens or damaged host cells.

According to recent animal models of ALS, the very first detectable pathophysiological changes are seen in the neuromuscular junctions and might be a sign of aberrant complement activation. ${ }^{5}{ }^{6}$ Such inappropriate activation of the complement cascades may participate in the initiation of the destruction of the neuromuscular junctions by an autoinflammatory mechanism. Furthermore, aberrant complement activation has been found in the proximity of the degenerating motor neurons during ALS progression. In addition, findings of complement activation in the blood and in the muscle tissue, as well as an unexplained erythrocyte cytotoxicity phenomenon found in the plasma of patients with ALS, suggest that the complement system could play an important role in the pathophysiology during the progression of ALS. ${ }^{4}$

To be able to develop an effective medical treatment, it is essential to study the pathogenesis and the pathophysiology of ALS. Since complement inhibition employing the terminal complement inhibitor ravulizumab now is entering a phase 3 clinical trial for the treatment of ALS, it is crucial to firmly establish how the complement system affects the progression of ALS (https://news.alexionpharma.com). Understanding the pathophysiology of the disease provides a tool to develop diagnostic methods such as biomarkers. This may optimise the diagnostic process ensuring a faster and more precise diagnosis. In addition, this could increase the knowledge of the heterogeneity of the ALS symptoms.

\section{Aim}

The overall, long-term objective of this project is to establish an ALS biobank to facilitate future basic ALS research. Under the assumption that ALS is an autoinflammatory disease, another aim of the project is to disclose whether complement activation plays an essential role in the pathophysiology of ALS. The protocol describes the design and data collection methods in detail in order to inform other researchers of the biobank.

\section{METHODS \\ Design}

This Danish national ALS biobank project is designed as a prospective, observational case-control study, based on the Standard Protocol Items: Recommendations for Interventional Trials guidelines. ${ }^{8}$ The biobank will be established to facilitate future studies. The first study comprises four substudies (substudy 1-4, table 1) of which substudy 1 and 2 are ongoing and the recruitment of participants for substudy 3 and 4 is planned to begin in the fall, 2020. Collection of biological material from patients with ALS for the longitudinal cohort study, substudy 3, is planned to continue as long as the included patients with ALS consent to lumbar puncture, blood sampling, and a short interview every 6 months, if possible.

\section{Patient and public involvement}

Patients or the public were not involved in the design, conduct, reporting or dissemination plans of this research.

\section{Participants}

The national ALS bio-bank comprises samples from three groups of patients: patients with ALS, neurologically healthy patients (healthy controls) and patients with neurological diseases other than ALS (neurological controls, NCs). All the participants included are above 18 years.

\section{Patients with ALS}

Patients referred to an ALS outpatient clinic at either of five Danish hospitals (table 2) due to symptoms suspected to be early symptoms of ALS are informed and invited to participate in the project during the standard clinical workup. If a patient at the end of the planned workup is diagnosed with either probable or definite ALS according to El Escorial Revised criteria ${ }^{9}$ and consents to inclusion, the patient is included in the project. Furthermore, all probable or definite patients with ALS who have already been permanently associated to an ALS outpatient clinic (post clinical investigation) and who accept to undergo an additional lumbar puncture are invited to be part of the project.

\section{Neurologically healthy controls}

Neurologically healthy patients scheduled for elective orthopaedic surgery under spinal anaesthesia are invited to participate.

\section{Neurological controls}

Patients admitted to hospital due to acute symptoms consistent with a subarachnoid haemorrhage are all subjected to a CT scan of the head. If this scan is without any signs of haemorrhage, an acute lumbar puncture will be carried out. We recruit participants for the NC group from this group of patients and the primary investigator performs the lumbar puncture. Furthermore, patients who are referred for a planned lumbar puncture as part of a clinical neurological investigation due to neurological symptoms are also recruited for the NC group.

\section{Exclusion criteria for the two control groups}

Subjects with motor neuron disease, chronic inflammatory or autoimmune diseases are excluded from the neurologically healthy control (NHC) group and the NC group.

\section{Settings}

Patients with ALS are recruited from five ALS outpatient clinics at five Danish neurological departments: Bispebjerg Hospital, Rigshospitalet (Glostrup) (both 
Table 1 Overview and the inclusion status of the four substudies

Substudy 1: a case-control study: haemolytic activity in ALS plasma

\begin{tabular}{|c|c|}
\hline Course of study & $\begin{array}{l}\text { Interviews. } \\
\text { Blood samples. } \\
\text { Separation of red blood cells and plasma. } \\
\text { Red blood cells incubated with blood type matched plasma } \\
\text { with or without inactivated complement system. } \\
\text { The degree of haemolysis is measured and compared. }\end{array}$ \\
\hline Subjects: aim/included so far & ALS: $25 / 25$ NC: $25 / 25$ NHC: $25 / 25$ \\
\hline \multicolumn{2}{|c|}{ Substudy 2: a case-control study: collection of material for biobank and profiling of the complement system } \\
\hline Course of study & $\begin{array}{l}\text { Interviews. } \\
\text { Blood samples and cerebrospinal fluid by lumbar puncture. } \\
\text { Biological material is prepared and freezed on site }\left(-20^{\circ} \mathrm{C}\right) \text {. } \\
\text { Transportation of samples to central biobank for storage } \\
\left(-80^{\circ} \mathrm{C}\right) \text {. } \\
\text { Profiling of the complement system comparing the three } \\
\text { groups. }\end{array}$ \\
\hline ubjects: aim/Included so fa & 100/61 NHC: $100 / 96$ \\
\hline
\end{tabular}

Substudy 3: a cohort study: collection of material for biobank and a study of the complement system over time

Course of study

The same course as in Substudy 2 repeated every 6 months

Subjects: aim/Included so far

ALS: 20/0 NC: - NHC: -

Substudy 4: pilot study: searching for complement activity in the neuromuscular junctions of patients with ALS

Course of study

- Interviews.

- Tru-cut biopsy from dominant lateral vastus muscle.

- Transportation of muscle biopsy to Department of Neuropathology, Hospitalise.

- Analysis of complement activity in muscle fibres and neuromuscular junctions.

Subjects: aim/Included so far

ALS: 10/0 NC: - NHC: -

ALS, amyotrophic lateral sclerosis; C, celsius; NC, neurological controls; NHC, neurologically healthy controls.

University of Copenhagen), Roskilde University Hospital, Odense University Hospital, and Aarhus University Hospital. NHCs are recruited at Gildhøj Private Hospital, Denmark. Controls from the neurological group are recruited at the neurological department at Rigshospitalet (Glostrup), Denmark. At each hospital, one local investigator is appointed. During sample collection, the primary investigator is present to obtain biological material from each patient and to process and freeze the samples using a portable 'mini-lab'. All data collected on case report forms are anonymised, gathered and stored in an audited, central, electronic database. All biological material is transported to the Department of Clinical Immunology at Rigshospitalet, Denmark to be stored in biobanking facilities.

\section{Data collection}

In patients with ALS and patients in the NC group, blood samples are drawn from a peripheral vein after the performance of the lumbar puncture. In NHCs, for logistical reasons, the blood samples are drawn before the lumbar puncture. All the samples are processed by the primary investigator at the respective inclusion site and are transported in a $-20^{\circ} \mathrm{C}$ freezer to a central biobank where they are stored at $-80^{\circ} \mathrm{C}$ (figure 1).

\section{Biological samples}

Blood samples

Venous blood samples are collected by the primary investigator or by a skilled clinical nurse. Blood is collected in sample 1: ethylenediaminetetraacetic acid (EDTA) blood tube $(8 \mathrm{~mL})$, sample 2: hirudin blood tubes $(8 \mathrm{~mL})$, sample 3: heparin lithium plasma tubes $(8 \mathrm{~mL})$, sample 4 : blood tube with clot activator for serum $(8 \mathrm{~mL})$, sample 5: PAXgene tube with RNAlater $(2.5 \mathrm{~mL})$. Samples 1-3 are centrifuged immediately at $2000 \mathrm{~g}$ for $10 \mathrm{~min}$. The supernatant (plasma) is aliquoted in small volumes into $1.5 \mathrm{~mL}$ tubes. The precipitate from sample 1 is aliquoted into $1.5 \mathrm{~mL}$ tubes. Sample 4 is kept for $45-60 \mathrm{~min}$ at room temperature and is then centrifuged at $2000 \mathrm{~g}$ for $10 \mathrm{~min}$. The supernatant (serum) is aliquoted in small volumes into $1.5 \mathrm{~mL}$ tubes. All samples are processed by the primary investigator. Subsequently, all the processed samples are transported in a $-20^{\circ} \mathrm{C}$ freezer and stored in a central biobanking facility at $-80^{\circ} \mathrm{C}$. Sample 5 is placed vertically at room temperature for $24-72$ hours, is frozen 
Table 2 Demographic data of the included patients with ALS in substudy 2

\begin{tabular}{|c|c|c|}
\hline Characterisation of the ALS group (n: 96) & $\mathbf{n}$ & $\%$ \\
\hline \multicolumn{3}{|l|}{ Inclusion site } \\
\hline Glostrup Hospital & 9 & 9 \\
\hline Bispebjerg Hospital & 33 & 34 \\
\hline Roskilde Hospital & 33 & 34 \\
\hline Odense Hospital & 16 & 17 \\
\hline Aarhus Hospital & 5 & 5 \\
\hline \multicolumn{3}{|l|}{ Age (median age: 67 ) } \\
\hline$<40$ & 3 & 3 \\
\hline $40-49$ & 12 & 13 \\
\hline $50-59$ & 13 & 14 \\
\hline $60-69$ & 28 & 29 \\
\hline$>70$ & 40 & 42 \\
\hline \multicolumn{3}{|c|}{ Age when first symptoms occurred (median age: 65) } \\
\hline$<40$ & 4 & 4 \\
\hline $40-49$ & 13 & 14 \\
\hline $50-59$ & 15 & 16 \\
\hline $60-69$ & 35 & 36 \\
\hline$>70$ & 29 & 30 \\
\hline \multicolumn{3}{|l|}{ Gender } \\
\hline Female & 40 & 42 \\
\hline Male & 56 & 58 \\
\hline
\end{tabular}

\section{ALS subtype I}

$\begin{array}{lrr}\text { Familial ALS } & 2 & 2 \\ \text { Sporadic ALS } & 94 & 98 \\ \text { ALS subtype II } & & \\ \text { Spinal ALS } & 60 & 63 \\ \text { Bulbar ALS } & 28 & 29 \\ \text { Both spinal and bulbar ALS } & 6 & 6 \\ \text { Truncal ALS } & 2 & 2\end{array}$

ALSFRS-R score on date collection day (median score: 37 ): Mild ALS symptoms (ALSFRS-R score >36) $49 \quad 51$ Moderate ALS symptoms $(24<$ ALSFRSR $33 \quad 34$ score $\leq 36$ )

Severe ALS symptoms (ALSFRS-R score $\leq 24) \quad 14 \quad 15$

Progression rate estimation ( $\Delta$ time*ALSFRS-R score on data collection day)

$\begin{array}{lll}\text { Slow progression rate } & 26 & 27 \\ \text { Medium progression rate } & 37 & 39 \\ \text { Aggressive progressive rate } & 33 & 34\end{array}$

Overall survival time from onset of symptoms (Database update: 2 May 2020)

\begin{tabular}{lll} 
Still alive (missing data) & 39 & 41 \\
Short survival time (0-2 years) & 22 & 23 \\
Medium survival time (2-4 years) & 25 & 26 \\
Long survival time (4+ years) & 10 & 10 \\
Cognitive impairments observed & & \\
Yes & 21 & 22 \\
\hline
\end{tabular}

Continued

\section{Table 2 Continued}

\begin{tabular}{lll}
\hline Characterisation of the ALS group (n: 96) & $\mathbf{n}$ & $\%$ \\
\hline No & 75 & 78 \\
Riluzole treatment on data collection day & & \\
Yes & 33 & 34 \\
No & 63 & 66 \\
\hline
\end{tabular}

ALS, Amyotrophic lateral sclerosis; ALSFRS-R, Amyotrophic Lateral Sclerosis Functional Rating Scale-Revised.

at $-20^{\circ} \mathrm{C}$ for $24-72$ hours, and is subsequently stored at $-80^{\circ} \mathrm{C}$.

\section{Cerebrospinal fluid samples}

On lumbar puncture, the first $1 \mathrm{~mL}$ of cerebrospinal fluid (CSF) obtained is discarded. Thereafter, $4-6 \mathrm{~mL}$ of CSF is obtained as feasible. Samples are immediately centrifuged at $2000 \mathrm{~g}$, and the supernatant is aliquoted into $1.5 \mathrm{~mL}$ tubes.

\section{Biobank facility}

The processed blood samples and the processed CSF samples will be stored in a biobank in a $-80^{\circ} \mathrm{C}$ freezer located at Laboratory of Molecular Medicine, Department of Clinical Immunology, Rigshospitalet, Copenhagen, Denmark.

Muscle biopsies (substudy 4)

After injection of local anaesthesia (lidocaine 2\%) one tru-cut biopsy is obtained containing $100 \mathrm{mg}$ of skeletal muscle tissue from the lateral vastus muscle. The biopsy is transported to the Department of Neuropathology, Rigshospitalet for further processing.

Base-line information

The following demographic information about the patients with ALS are collected: age, gender, subtype of disease, current stage of disease, $\mathrm{T}_{0}$ (defined as the month

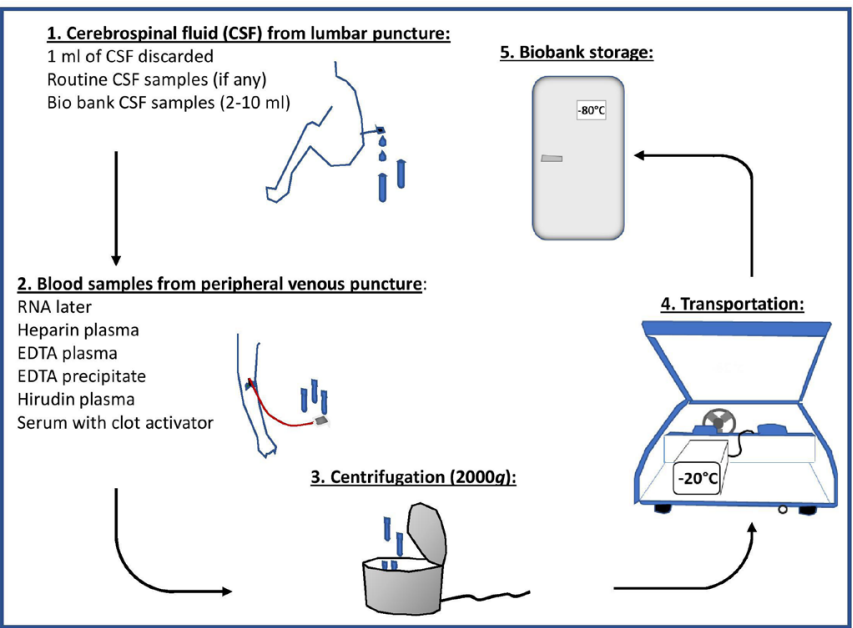

Figure 1 Collection of biological material.EDTA, ethylenediaminetetraacetic acid; g, therelative centrifugal force; RNA, ribonucleidacid 


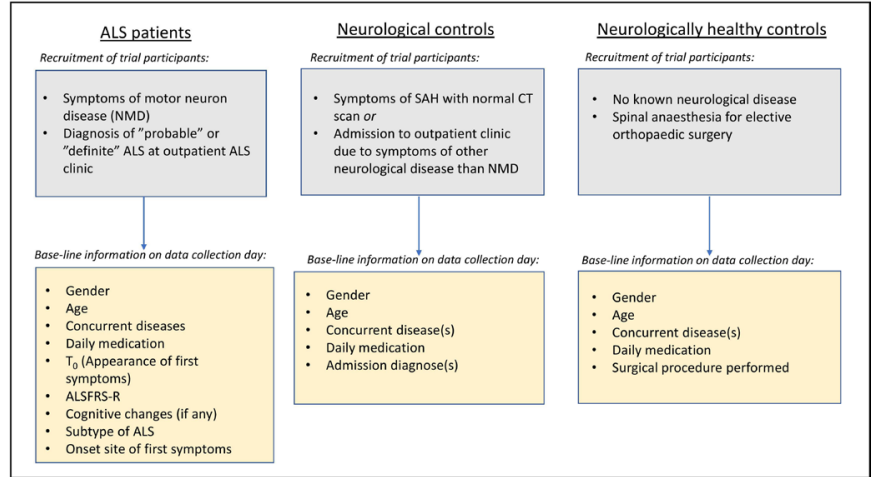

Figure 2 Inclusion and baseline registration. ALS, amyotrophic lateral sclerosis; ALSFRS-R, Amyotrophic Lateral Sclerosis Functional Rating Scale-Revised; NMD, neuromuscular disease; $\mathrm{SAH}$, subarachnoid haemorrhage; $\mathrm{T}_{0}$, time of first symptoms (month and year).

and year of the first subjective symptoms that later led to the diagnosis of either probable ALS or definite ALS), treatment with riluzole, daily medication and observed cognitive changes. Data are pseudonymised and entered into an Excel database only accessible for A-LK, PG and $\mathrm{KM}$ and the database is updated, stored, and logged in accordance with Danish law and regulations set by the Danish Data Protection Agency. An interview of all the patients with ALS, based on the questionnaire ALS functional Rating Scale-Revised (ALSFRS-R), ${ }^{10}$ is conducted by the primary investigator on the day of lumbar puncture and blood sampling (data collection day) and the score is noted in the database (figure 2).

\section{Clinical phenotyping}

The subtype of ALS is noted: sALS/fALS and the subtype of spinal, bulbar or truncal ALS. Furthermore, it is noted whether the ALS specialists observe any sign of cognitive impairment. ALSFRS-R score, estimated on the day of data collection, is noted. Patients are age categorised into age groups. Time of the first appearance of symptoms $\left(\mathrm{T}_{0}\right)$ is noted and the clinical status on data collection day is categorised.

\section{Analysis}

\section{Complement measurements}

The concentrations of complement components (ficolin-1, ficolin-2, ficolin-3, collectin-11, pentraxin 3 (PTX3), mannose-binding lectin (MBL), MBL/ficolin/ CL-associated serine protease 3 (MASP-3), MBL/ficolin/ Cl-associated protein 1 (MAP-1), complement activation products (C4c, C3bc, sC5b-9) as well as complement activation potentials (measured as activation on pathway-specific ligands: human serum albumin/ antihuman serum albumin immune complexes for classical pathway activation; lipopolysaccharide for alternative pathway activation; mannan for MBL-mediated lectin pathway activation; and acetylated bovine serum albumin for ficolin-mediated lectin pathway activation) are measured by ELISA in plasma and CSF samples. The
ELISA experiments are performed at the Laboratory of Molecular Medicine, Rigshospitalet, using specific monoclonal antibodies as previously described. ${ }^{11-23}$ Total serum concentrations of C4 and C3 are quantified by an automated turbidimetric protein analyser (SPAPLUS, The Binding Site group LDT, Birmingham, UK) where sheep polyclonal antibodies against either human C3c or human C4 are applied (The Binding Site group LDT, Birmingham, UK).

\section{Cytokine measurement}

Selected cytokines as well as acute phase reactants will be analysed with a multiplex sandwich immunoassay with electrochemiluminescence: plates, precoated with capture antibodies for the cytokines, are incubated with plasma samples. Subsequently, detection antibodies are put in the wells and then the plates are incubated. After washing, the detection levels are measured.

\section{RNA expression and proteomics studies}

Full blood is obtained in PAXgene Blood RNA tubes with RNA later, an RNA stabilising buffer, for future isolation of RNA and gene expression profiling. At that point in time, we will be able to conduct proteomic analysis simultaneously as well as using the same samples of whole blood preserved in RNA later.

\section{Neuropathological studies}

For immunofluorescence staining, sections of ALS muscle biopsies from the lateral vastus muscle is air-dried and fixed in $4 \%$ paraformaldehyde at $-20^{\circ} \mathrm{C}$. Slides are washed in phosphate-buffered saline (PBS), permeabilised in PBS $/ 0.2 \%$ TritonX and blocked using PBS $/ 5 \%$ fetal calf serum $/ 0.2 \%$ TritonX. The sections are stained with primary antibodies directed against complement components and regulators (anti-C3c, anti-C1q, anti-C4c, anti-C5b-9 and anti-CD59), followed by incubation with fluorescence marked secondary antibodies. The motor end plates are visualised by incubating with Alexa 488 conjugated antibungarotoxin, which binds to postsynaptic acetylcholine receptors on the muscle fibres, thus visualising the end plates. After staining, the sections are washed in PBS, air dried and mounted on slides. The muscle sections are analysed for complement staining and colocalisations with motor end plates using confocal microscopy. Each motor end plate identified on the surface of a muscle fibre will be counted using automated software, and the length of the end plates will be measured in the ALS muscle biopsies. The size of end plates will be measured and the number of immunoreactive areas per section will be scored.

\section{Outcome measures, sample size and statistics for each substudy}

Substudy 1

\section{Primary and explorative outcomes}

The primary outcome is the difference in haemolytic activity of plasma against healthy red erythrocytes as measured by absorbance between patients with ALS and 
NHCs. The difference in haemolytic activity between patients with ALS and NCs is an exploratory outcome.

\section{Sample size}

Overgaard et al measured the haemolytic activity after incubation of healthy as well as ALS erythrocytes in both healthy plasma and ALS plasma. They described a mean difference of 0.20 (SE 0.052 in the group of patients with ALS, number 20, SD 0.22) in the absorbance $(415 \mathrm{~nm}$, incubation: 5 hours) between ALS group and group of healthy controls. ${ }^{24}$ At $\alpha=0.05$ and $\beta=0.20$, we need to include 21 participants in each group. Taking into account possible dropouts, failed technical tests, inclusion of 25 persons per group is planned.

\section{Statistical analysis}

The haemolytic activity in the three groups (patients with ALS, NCs, NHCs) will be compared using one-way analysis of variance (ANOVA) followed by t-tests to pinpoint differences between groups. cut-off values and predictive values will be calculated using receiver operating characteristic (ROC) curves.

\section{Substudy 2}

Primary and explorative outcomes

The primary outcome is the activation potential of the ficolin-mediated lectin pathway. The activation potential of the classical pathway, the alternative pathway and the MBL-mediated lectin pathway are exploratory outcomes.

\section{Sample size}

The number of subjects in each group is calculated using an $\alpha=0.05$ and $\beta=0.20$. We compare the complement activation potential of three equal-sized groups. In healthy subjects, the complement activation potential is $100 \%$ with a normal area ranging from $50 \%$ to $150 \%$, and the prevalence of subjects with a low complement activation potential (under 50\%) is under 10\%. If 100 subjects are included in each group, it will be possible to detect statistically significant differences between the groups corresponding to an OR of 2.3, which would correspond to $20 \%$ of patients with ALS having a low complement activation potential caused by increased complement activity. ${ }^{25}$

\section{Statistical analysis}

The concentration of complement markers and the complement activation potential will be compared between patients with ALS and the two control groups using stepwise ANOVA followed by t-tests, Bonferronicorrected as appropriate. If necessary, we will logtransform the data to ensure a Gaussian distribution. The covariates, that will be used for the stepwise ANOVA to test for differences between the three groups, are subject category (patient with ALS, NHC or NC), gender and age.

The patients with ALS will be described and categorised by the covariates as illustrated in table 1 . We will analyse the covariates and the response variables by doing stepwise, one-way ANOVA to test for differences between the ALS subtypes.
Cut-off values and predictive values are calculated using ROC curves.

\section{Substudy 3}

\section{Primary and explorative outcomes}

The primary outcome measure is the change in the plasma/CSF concentration ratio of ficolin-3 over time in patients with rapid progression compared with those with slow progression. The concentrations in plasma and CSF of ficolin-1, ficolin-2, ficolin-3, collectin-11, PTX3, MASP-3, MBL, MAP-1, C4c, C3bc, sC5b-9 as well as the plasma/CSF concentration ratios of ficolin-1, ficolin-2, collectin-11, PTX3, MASP-3, MBL, MAP-1, C3bc, C4c and sC5b-9 are exploratory outcomes.

\section{Sample size}

To our knowledge, no prior studies describe the activity of the complement system in patients with ALS over time. This is hence a pilot study for which no sample size calculation has been made. We will include 20 patients with ALS.

\section{Statistical analysis}

Analyses are carried out as described for substudy 2. In addition, we will conduct linear regression analyses with the levels of complement proteins as the dependent variable and time since onset of disease, gender, age and subtype of ALS as explanatory variables.

\section{Substudy 4}

\section{Primary and explorative outcomes}

The primary outcome is the presence of any marker of the complement system in the neuromuscular junction as visualised by confocal microscopy of muscle biopsies from patients with ALS. These putative observations will be compared against an existing normative sample of muscle biopsies.

\section{Sample size}

This is a hypothesis-generating study as the presence of complement activity in living patients with ALS have not been described previously; therefore, no sample size calculation has been made. We will include 10 patients with ALS.

\section{Statistical analysis}

Complement depositions in the muscle fibres and in particular in the neuromuscular junctions are described qualitatively. The samples are scored by an investigator, who is blinded to clinical information, into either 'normal', 'light degree' or 'Severe degree' of changes. These assessments will be compared with $2 \mathrm{x} \mathrm{K}$ tables and non-parametric statistics.

\section{DISCUSSION}

ALS is a rapidly progressive, fatal disease. Despite the aggressive course and devastating consequences, there is currently no effective treatment of ALS. 
This manuscript describes the generation of a Danish ALS biobank that will hopefully provide a starting point for future national ALS research. With the present project, a substantial amount of biological material from patients suffering from ALS will be obtained. We hope that the project will bring ALS research a significant step forward, will inspire other groups to start similar projects regarding this and other rare diseases, and hence will enable future basic biobank research within this challenging field.

The generation of this biobank was inspired by the hypothesis that the innate immune system plays a significant role in the pathogenesis or the pathophysiology of ALS. With a specific aim to profile the complement system in patients with ALS, it became important to ensure that the two control groups do not include patients with diseases with neither primary nor secondary complement activation even if this means that the two control groups will not be comparative in age and gender.

Several initiatives have conducted multicentre, interventional clinical trials and established large ALS biobanks. ${ }^{26}$ Continuing the collection of biological material from patients with this rare disease will maintain and expand the capacity to study the increasing number of hypotheses generated by the spatiotemporal studies conducted on animal ALS models. ${ }^{562728}$ With the present protocol, we intend to obtain biological material from patients with ALS starting in a relatively early phase of clinical disease. Furthermore, patients with ALS are invited to participate in the cohort study (substudy 3) which will add a spatiotemporal aspect to the biobank.

All Danish citizens are registered in the National Patient Registry (DNPR) which is one of the oldest complete, national patient registries in the world. DNPR is linkable on an individual level with other clinical and administrative registries. ${ }^{29}$ Hence, this poses a unique possibility to conduct registry-based research combined with data from this ALS biobank in future studies.

More than 20 genetic mutations have been associated with ALS, the most well-known and well-researched mutations being the SOD1, FUS, TARDBP and C9ORF72 genes. ${ }^{30}$ Even though ALS seems to have a multifactorial aetiology, genetic mutations may play a pivotal role in the pathogenesis of ALS. Our planned substudies do not include genetic analysis. However, future genetic studies will be possible as biological material in the form of EDTA precipitate will be secured for the biobank.

\section{TRIAL LIMITATIONS}

All patients have been interviewed by the same person preventing inter-rater variability. The possibility of reporting bias is not prevented, however, as the interviewer might subtly change the way of questioning over time, even though they are using the same questionnaire for all patients. Additionally, the $\mathrm{T}_{0}$ can be difficult for the patients to define and remember and lead to imprecise or even incorrect answers. ${ }^{31}$
Retrospectively, it has been common procedure to include patients with probable or definite ALS in clinical ALS research projects. Some patients have slowly progressing symptoms of a motor neuron disease which initially do not meet the criteria of probable or definite ALS as the upper and lower motor neurons are not yet both affected. Even so, the symptoms may progress in which case the patient will eventually meet the criteria of probable or definite ALS. With the inclusion criteria stated for the present protocol, we realise that we may miss inclusion of this subset of (possible) patients with ALS and hence the opportunity to observe the initial phase of such patients. ${ }^{32}$

Information about cognitive impairment/changes registered in the electronic patient file has been entered into the database. However, less severe symptoms in some cases of cognitive impairment might be overlooked by the clinicians, and ideally, patients should undergo standardised, neuropsychological testing. However, as it was not within the scope of this project to ensure neuropsychological testing of the patients with ALS, we elected to rely on the practice of the clinical inclusion sites, which unfortunately does not always comprise testing, regardless of cognitive changes.

Substudy 4 is a pilote study based on tru-cut skeletal muscle biopsies from 10 patients with ALS. Since only one biopsy is obtained from each patient, this is a rather small amount of tissue in particular for a neuromuscularjunction study. We chose this option as a compromise given the pilot nature of the study, to inflict as little pain as possible on the patient.

The NHCs in the biobank and hence also for the four substudies are included if they have no neurological symptoms based only on the anamnesis. However, to ensure that these are neurologically healthy, one could argue that all the NHCs should have a clinical neurological examination performed as well as an MRI scan of the CNS before inclusion.

\section{Author affiliations}

${ }^{1}$ Neuroanaesthesiology, The Neuroscience Centre, Rigshospitalet, Copenhagen, Denmark

${ }^{2}$ Laboratory of Molecular Medicine, Department of Clinical Immunology Section 7631, Diagnostic Centre, Rigshospitalet, Copenhagen, Denmark

${ }^{3}$ Neurology, The Neuroscience Centre, Rigshospitalet, Glostrup, Denmark ${ }^{4}$ Institute of Clinical Medicine, Faculty of Health and Medical Sciences, University of Copenhagen, Copenhagen, Denmark

Acknowledgements The authors are most grateful towards all the patients that consent to participate in this study. Furthermore, we wish to thank clinicians and researchers at the neurological departments that are inclusion sites for the substudies, as well as Gildhøj Private Hospital, which is the inclusion site for neurologically healthy patients.

Contributors KSO, SWP, AØL, KM, PG and A-LK conceived and designed the study. A-LK drafted the protocol, and A-LK, KSO, SWP, AØL, KP, KM and PG revised the protocol for important intellectual content. A-LK, AØL, KSO and KM implemented the study at the clinical departments. A-LK drafted the manuscript. All authors revised the manuscript for important intellectual content and assume responsibility for the final version. 
Funding This work was funded by Aase and Ejnar Danielsen's Foundation, The Jascha Foundation, The Danish Research Foundation of Independent Research (DFF-6110-00489), and The Danish Heart Foundation (16-R107-A6650-22966).

\section{Competing interests None declared.}

Patient consent for publication Not required.

Provenance and peer review Not commissioned; externally peer reviewed.

Open access This is an open access article distributed in accordance with the Creative Commons Attribution Non Commercial (CC BY-NC 4.0) license, which permits others to distribute, remix, adapt, build upon this work non-commercially, and license their derivative works on different terms, provided the original work is properly cited, appropriate credit is given, any changes made indicated, and the use is non-commercial. See: http://creativecommons.org/licenses/by-nc/4.0/.

Author note A steering committee will be established with representatives from clinical as well as basic ALS research groups in order to ensure easy access to the collected biological material for future, state-of-the-art ALS research projects.

ORCID iD

Anne-Lene Kjældgaard http://orcid.org/0000-0002-7197-8298

\section{REFERENCES}

1 Phukan J, Pender NP, Hardiman O. Cognitive impairment in amyotrophic lateral sclerosis. Lancet Neurol 2007;6:994-1003.

2 Chiò A, Logroscino G, Traynor BJ, et al. Global epidemiology of amyotrophic lateral sclerosis: a systematic review of the published literature. Neuroepidemiology 2013;41:118-30.

3 Philips T, Rothstein JD. Rodent models of amyotrophic lateral sclerosis. Curr Protoc Pharmacol 2015;69:5.67.1-5.67.21.

4 Kjældgaard A-L, Pilely K, Olsen KS, et al. Amyotrophic lateral sclerosis: the complement and inflammatory hypothesis. Mol Immunol 2018:102:14-25.

5 Bahia El Idrissi N, Bosch S, Ramaglia V, et al. Complement activation at the motor end-plates in amyotrophic lateral sclerosis. $J$ Neuroinflammation 2016;13:72

6 Heurich B, El Idrissi NB, Donev RM, et al. Complement upregulation and activation on motor neurons and neuromuscular junction in the SOD1 G93A mouse model of familial amyotrophic lateral sclerosis. $J$ Neuroimmunol 2011;235:104-9.

7 Parker SE, Hanton AM, Stefanou SN, et al. Revisiting the role of the innate immune complement system in ALS. Neurobiol Dis 2019;127:223-32.

8 Chan A-W, Tetzlaff JM, Gøtzsche PC, et al. Spirit 2013 explanation and elaboration: guidance for protocols of clinical trials. $B M J$ 2013;346:e7586.

9 Brooks BR, Miller RG, Swash M, et al. El Escorial revisited: revised criteria for the diagnosis of amyotrophic lateral sclerosis. Amyotroph Lateral Scler Other Motor Neuron Disord 2000;1:293-9.

10 Cedarbaum JM, Stambler N, Malta E, et al. The ALSFRS-R: a revised ALS functional rating scale that incorporates assessments of respiratory function. BDNF ALS Study Group (phase III). J Neurol Sci 1999;169:13-21.

11 Bayarri-Olmos R, Kirketerp-Moller N, Pérez-Alós L, et al. Development of a quantitative assay for the characterization of human Collectin-11 (CL-11, CL-K1). Front Immunol 2018;9:2238.

12 Bastrup-Birk S, Skjoedt M-O, Munthe-Fog L, et al. Pentraxin-3 serum levels are associated with disease severity and mortality in patients with systemic inflammatory response syndrome. PLoS One 2013;8:e73119.
13 Skjoedt M-O, Palarasah Y, Munthe-Fog L, et al. MBL-associated serine protease- 3 circulates in high serum concentrations predominantly in complex with ficolin-3 and regulates ficolin-3 mediated complement activation. Immunobiology 2010;215:921-31.

14 Skjoedt M-O, Hummelshoj T, Palarasah Y, et al. Serum concentration and interaction properties of MBL/ficolin associated protein-1. Immunobiology 2011;216:625-32.

15 Seelen MA, Roos A, Wieslander J, et al. Functional analysis of the classical, alternative, and MBL pathways of the complement system: standardization and validation of a simple ELISA. J Immunol Methods 2005;296:187-98.

16 Hein E, Honoré C, Skjoedt M-O, et al. Functional analysis of ficolin-3 mediated complement activation. PLoS One 2010;5:e15443.

17 Pilely K, Skjoedt M-O, Nielsen C, et al. A specific assay for quantification of human $\mathrm{C} 4 \mathrm{c}$ by use of an anti-C4c monoclonal antibody. J Immunol Methods 2014;405:87-96.

18 Garred P, Mollnes TE, Lea T. Quantification in enzyme-linked immunosorbent assay of a C3 neoepitope expressed on activated human complement factor C3. Scand J Immunol 1988;27:329-35.

19 Mollnes TE, Lea T, Frøland SS, et al. Quantification of the terminal complement complex in human plasma by an enzyme-linked immunosorbent assay based on monoclonal antibodies against a neoantigen of the complex. Scand J Immunol 1985;22:197-202.

20 Garred P, Madsen HO, Kurtzhals JA, et al. Diallelic polymorphism may explain variations of the blood concentration of mannan-binding protein in Eskimos, but not in black Africans. Eur J Immunogenet 1992;19:403-12.

21 Munthe-Fog L, Hummelshøj T, Hansen BE, et al. The impact of FCN2 polymorphisms and haplotypes on the ficolin-2 serum levels. Scand $\mathrm{J}$ Immunol 2007;65:383-92.

22 Munthe-Fog L, Hummelshøj T, Ma YJ, et al. Characterization of a polymorphism in the coding sequence of FCN3 resulting in a ficolin-3 (Hakata antigen) deficiency state. Mol Immunol 2008;45:2660-6.

23 Munthe-Fog L, Hummelshoj T, Honoré C, et al. Variation in FCN1 affects biosynthesis of ficolin-1 and is associated with outcome of systemic inflammation. Genes Immun 2012;13:515-22.

24 Overgaard K, Werdelin L, Sørensen $\mathrm{H}$, et al. Cytotoxic activity in plasma from patients with amyotrophic lateral sclerosis. Neurology 1991;41:925-7.

25 Armitage PBG. Statistical methods for medical researchers. Blackwell, 1994.

26 Sherman AV, Gubitz AK, Al-Chalabi A, et al. Infrastructure resources for clinical research in amyotrophic lateral sclerosis. Amyotroph Lateral Scler Frontotemporal Degener 2013;14:53-61.

27 Wang H, Ricklin D, Lambris JD. Complement-activation fragment C4a mediates effector functions by binding as untethered agonist to protease-activated receptors 1 and 4. Proc Natl Acad Sci U S A 2017:114:10948-53.

28 Wang HA, Lee JD, Lee KM, et al. Complement C5a-C5aR1 signalling drives skeletal muscle macrophage recruitment in the hSOD1 ${ }^{\mathrm{G} 93 \mathrm{~A}}$ mouse model of amyotrophic lateral sclerosis. Skelet Muscle 2017;7:10.

29 Schmidt M, Schmidt SAJ, Sandegaard JL, et al. The Danish national patient registry: a review of content, data quality, and research potential. Clin Epidemiol 2015;7:449-90.

30 Nowicka N, Juranek J, Juranek JK, et al. Risk factors and emerging therapies in amyotrophic lateral sclerosis. Int J Mol Sci 2019;20. doi:10.3390/ijms20112616. [Epub ahead of print: 28 May 2019].

31 Taylor-Rowan M, Wilson A, Dawson J, et al. Functional assessment for acute stroke trials: properties, analysis, and application. Front Neurol 2018:9:191-91.

32 Al-Chalabi A, Hardiman O, Kiernan MC, et al. Amyotrophic lateral sclerosis: moving towards a new classification system. Lancet Neurol 2016;15:1182-94. 